\title{
Estudio analítico de la composición en ácidos grasos de mostaza y salsas de mostaza
}

\author{
Por E. López Argüello, C. Barrera Vázquez y N. Bosch Bosch \\ Departamento de Nutrición y Bromatología II: Bromatología. Facultad de Farmacia. \\ Universidad Complutense de Madrid 28040 - Madrid. España.
}

\section{RESUMEN}

Estudio analítico de la composición en ácidos grasos de mostaza y salsas de mostaza.

Se determinaron, por cromatografía gaseosa, el perfil de los ácidos grasos en mostaza (semilla y harina), salsas de mostaza con grano y salsas finas de mostaza con el objeto de caracterizarlas en relación con la semilla base. El ácido erúcico fue el más abundante en la mostaza y en las salsas finas $(27,7 \%$ y $24,6 \%$ del contenido total de ácidos grasos), seguido de los ácidos oléico $(20,0 \%$ y $21,1 \%)$ y linoleico (16,3 y $16.2 \%)$. En las salsas de mostaza con grano el ácido graso mayoritario fue el linolénico $(21,4 \%)$ seguido de los ácidos linoleico $(20,3 \%)$ y oléico $(19,3 \%)$. Mediante los análisis de correlación y discriminante las muestras son agrupadas y clasificadas. Los ácidos grasos han demostrado ser parámetros válidos para el análisis del control de calidad de las salsas de mostaza.

PALABRAS-CLAVE: Acido graso - Cromatografía gaseosa Mostaza - Salsa de mostaza.

\section{SUMMARY}

Analytical study of the composition of fatty acid of mustard and mustard sauces.

In order to characterise mustard (whole seed or flour), mustard sauces with grain and mustard sauces in relation to their base seed the concentrations of fatty acid were determinated by gas chromatography (GC). Erucic acid was the most abundant fatty acid component in mustard and mustard sauces $(27,7 \%$ and $24,6 \%$ of total fatty acid content), followed by oleic acid $(20,0 \%$ and $21,1 \%)$ and linolenic acid $(16,3 \%$ and $16.2 \%)$. The major acid in mustard sauces with grain was linolenic $(21,4 \%)$, followed by linoleic acid $(20,3 \%)$ and oleic acid $(19,3 \%)$. By means of correlation and discriminant analysis the samples have been grouped and classified. It is shown that fatty acid are usefull parameters for quality control analysis of mustard sauces.

KEY-WORDS: Fatty acid - Gas chromatography - Mustard Mustard sauce.

\section{INTRODUCCIÓN}

Las semillas de mostaza, Brassica nigra (mostaza negra) y Sinapis alba (mostaza blanca), son especias utilizadas por su aroma y sabor picante. Las semillas se emplean en ensaladas, mientras que la mostaza preparada (salsas), que es una mezcla de mostaza con sal, vinagre, especias y otros condimentos, es utilizada como aderezo en sandwiches, salchichas y carnes. La harina de mostaza se emplea en la preparación de carnes, pescados y otras salsas.

El objetivo de este trabajo consiste en conocer la composición en ácidos grasos de la mostaza y salsas de mostaza, con el fin de caracterizar estos productos en relación con su semilla base, y poder evaluar la participación de ésta en la elaboración de estas salsas, así como averiguar la posible adición de algún tipo de aceite vegetal debido al empleo de otras especias y condimentos distintos de la mostaza.

\section{PARTE EXPERIMENTAL}

Las muestras objeto del presente estudio fueron mostazas (semillas o harinas), salsas de mostaza con grano y salsas finas de mostaza adquiridas en el mercado español. Excepto una muestra de semilla, que fue adquirida en Sri Lanka, todas las demás fueron preparadas o envasadas en España y en otros países de la Unión Europea (Francia, Inglaterra y Alemania).

Las muestras fueron agrupadas mediante el sistema que se indica a continuación:

Grupo 1: Mostaza (semilla, harina): 6 muestras. Grupo 2: Salsas de mostaza con grano: 6 muestras. Grupo 3: Salsa finas de mostaza: 34 muestras.

Una vez obtenido el extracto etéreo por el método de Soxhlet (Pearson, 1981), empleando éter etílico como disolvente, se realiza la metilación de los ácidos grasos empleando metilato sódico y metanol en ácido clorhídrico. A continuación, los ésteres metílicos formados se extraen con hexano y los extractos se lavan con agua hasta la desaparición total del ácido. Los líquidos decantados se deshidratan con sulfato sódico anhidro, y se elimina el disolvente utilizando un evaporador rotatorio al vacío bajo corriente de nitrógeno. Posteriormente, los ésteres metílicos se emplean para estudiar la composición en ácidos grasos de la mostaza y sus salsas mediante cromatografía gaseosa (Ministerio de Sanidad y Consumo, 1985). 
El instrumental empleado y las condiciones de trabajo para el análisis de ácidos grasos en mostazas por cromatografía gaseosa son las siguientes:

Cromatógrafo de gases: Perkin-Elmer, modelo Sigma 3B.

Detector de ionización de llama.

Columna de acero inoxidable de $2 \mathrm{~m}$ por 1/8".

Fase estacionaria: Dietilenglicolsuccinato al 15\% (DGS).

Soporte: Chromosorb W-AW; 80/100 mallas.

Gas portador: Nitrógeno; flujo: $30 \mathrm{ml} / \mathrm{min}$.

Temperaturas: Columna $195^{\circ} \mathrm{C}$. Inyector $260^{\circ} \mathrm{C}$. Detector $200^{\circ} \mathrm{C}$.

Registrador-Integrador: Perkin-Elmer, modelo Sigma 15.

La identificación de los ácidos grasos se realizó a partir de una solución patrón preparada por disolución en hexano de los correspondientes ésteres metílicos comercializados individualmente (Sigma Chemical, Co.).

Tabla 1

Acidos grasos característicos de las mostazas (\% del contenido total)

\begin{tabular}{cccc}
\hline & $\begin{array}{c}\text { Mostaza } \\
(\text { semilla y harina) } \\
(\mathrm{n}=6)\end{array}$ & $\begin{array}{c}\text { Salsas de } \\
\text { mostaza con grano } \\
(\mathrm{n}=6)\end{array}$ & $\begin{array}{c}\text { Salsas finas } \\
\text { de mostaza } \\
(\mathrm{n}=34)\end{array}$ \\
\hline Acido graso & $\mathrm{x}_{ \pm}$on -1 & $\mathrm{x}_{ \pm}$on -1 & $\mathrm{x} \pm$ on \\
\hline $16: 0$ & $2,83 \pm 0,17$ & $3,44 \pm 0,25$ & $3,95 \pm 1,29$ \\
$16: 1$ & $0,32 \pm 0,10$ & $0,37 \pm 0,04$ & $0,48 \pm 0,20$ \\
$18: 0$ & $1,44 \pm 0,34$ & $1,60 \pm 0,09$ & $1,86 \pm 0,96$ \\
$18: 1$ & $20,0 \pm 1,68$ & $19,3 \pm 1,75$ & $21,1 \pm 1,77$ \\
$18: 2$ & $15,3 \pm 4,16$ & $20,3 \pm 1,20$ & $15,9 \pm 4,78$ \\
$18: 3$ & $16,3 \pm 1,95$ & $21,4 \pm 7,96$ & $16,2 \pm 1,45$ \\
$20: 1$ & $11,4 \pm 0,94$ & $11,0 \pm 1,58$ & $10,7 \pm 0,72$ \\
$20: 2$ & $0,99 \pm 0,34$ & $1,01 \pm 0,13$ & $0,81 \pm 0,32$ \\
$22: 0$ & $0,75 \pm 0,20$ & $0,58 \pm 0,08$ & $0,63 \pm 0,08$ \\
$22: 1$ & $27,7 \pm 5,84$ & $18,5 \pm 2,85$ & $24,6 \pm 5,21$ \\
$24: 0$ & $0,29 \pm 0,08$ & $0,22 \pm 0,03$ & $0,27 \pm 0,06$ \\
\hline
\end{tabular}

Los resultados se han expresado en porcentaje del contenido total de los ácidos grasos (Tabla I).

Con el fin de lograr una mejor interpretación de los datos se han realizado el análisis discriminante y el de correlación lineal (Lebart, 1985; STSC, 1986).

\section{RESULTADOS Y DISCUSIÓN}

El contenido en extracto etéreo ha oscilado entre: $27,0-40,5 \%$ en las mostazas; $9,16-17,1 \%$ en las salsas de mostaza con grano y 4,21-19,8 \% en las salsas finas de mostaza de mostaza. Cuando se trata de salsas, se ha podido comprobar que en sus etiquetas no se indica la adición de ninguna grasa, por lo que se deduce que este residuo de carácter lipídico procede de la mostaza incorporada y de otros productos como son los aditivos solubles en éter como el E-471 (mono y diglicéridos de ácidos grasos) empleados como emulgentes en la industria alimentaria.

El principal ácido graso de la mostaza, característico de las crucíferas, es el erúcico (C22:1) (FAO, 1991); en el presente trabajo se obtuvo una concentración media, para este ácido graso de 27,7 $\pm 5,24 \%$ (\% del contenido total de ácidos grasos) (Tabla I). Los resultados obtenidos en este estudio en relación al erúcico son inferiores a los apartados por la FAO (1991), Sindhu et al., (1993) y se encuentran dentro del intervalo indicado por Karleskind (1992).

Otros ácidos grasos predominantes fueron el oléico $(20 \pm 1,68 \%)$, linolénico $(16,3 \pm 1,95 \%)$, linoléico $(15,3 \pm 4,16 \%)$ y eicosenoico $(11,4 \pm 0,94 \%)$, en proporciones semejantes a las aportadas por Wolff (1968) para los ácidos oléico y linoléico (Tabla I).

Las salsas finas de mostaza presentan el mismo perfil de ácidos grasos que las mostazas, y si se exceptúa al ácido erúcico, el resto de los ácidos se presentan en concentraciones similares en ambos grupos.

En cinco de las seis salsas de mostaza con grano los ácidos grasos predominantes fueron: linoléico, oléico y erúcico; sin embargo, una de las muestras presenta elevado contenido de ácido linolénico $(37,7 \%)$ y bajo de ácido erúcico (12,8\%), lo que puede ser indicativo del empleo de alguna nueva variedad de mostaza de bajo contenido en este ácido graso (Tabla I).

Además, se deduce que del total de ácidos grasos que forman parte de los tres grupos de mostazas, los que se encuentran en mayor proporción son los insaturados, de los cuales los que predominan son los monoinsaturados, que oscilan entre $49,1 \%$ en las salsas de mostaza con grano y $59,4 \%$ en la mostaza. El contenido de ácidos grasos saturados está comprendido entre $5,32 \%$ y $6,71 \%$ para la mostaza y las salsas finas de mostaza, respectivamente.

\subsection{Estudio estadístico}

En la realización del análisis discriminante se han tenido presentes todos los ácidos grasos estudiados. En la matriz de clasificación se aprecia que no todas las muestras están correctamente clasificadas. Una muestra correspondiente al grupo 1 (mostaza sólida) se clasifica como perteneciente al grupo 2 (salsas de mostaza con grano); otra de las muestras correspondiente a salsas de mostaza con grano se clasifica como perteneciente al grupo salsas finas de mostaza de mostaza; y, por último, de las treinta y cuatro salsas finas de mostaza, cuatro se clasifican como pertenecientes al grupo salsas con grano y otra al grupo mostazas sólidas (Tabla II). 
Tabla II

Matriz de clasificación

\begin{tabular}{ccccc}
\hline & $\begin{array}{c}\text { Porcentaje de } \\
\text { clasificación } \\
\text { correcto }\end{array}$ & \multicolumn{3}{c}{ Número de muestras en cada grupo } \\
\cline { 3 - 5 } & 83,33 & 5 & $\begin{array}{c}\text { Salsas de } \\
\text { mostaza con grano }\end{array}$ & $\begin{array}{c}\text { Salsas finas de } \\
\text { mostaza }\end{array}$ \\
\hline 1 & 83,33 & 0 & 1 & 0 \\
2 & 83,33 & 5 & 1 \\
3 & 85,29 & 1 & 4 & 29 \\
\hline
\end{tabular}

1: Mostaza (semilla y harina).

2: Salsas de mostaza con grano.

3: Salsas finas de mostaza.

Los coeficientes de las funciones discriminantes (Tabla III) permiten representar gráficamente todas

Tabla III

Coeficientes de las funciones discriminantes

\begin{tabular}{ccc}
\hline Variable & \multicolumn{2}{c}{ Coeficientes } \\
\hline $16: 0$ & $-2,16850$ & $-0,94789$ \\
$16: 1$ & $-0,39894$ & $-0,21328$ \\
$18: 0$ & 2,27477 & 0,84292 \\
$18: 1$ & 0,90252 & 0,16943 \\
$18: 2$ & 0,02546 & $-0,00029$ \\
$18: 3$ & 0,30230 & 0,64910 \\
$20: 1$ & $-0,75701$ & 0,29480 \\
$20: 2$ & 2,13265 & 0,12814 \\
$22: 0$ & $-0,08013$ & $-0,17827$ \\
$22: 1$ & 1,55122 & $-0,30098$ \\
$24: 0$ & 0,15999 & $-0,28041$ \\
\hline
\end{tabular}

las muestras. En la Figura 1 se refleja la posición de los tres grupos de muestras respecto a las funciones discriminantes primera y segunda. De ello se deduce que la mostaza se diferencia de las salsas en relación con la función discriminante primera; sin embargo, la segunda función discriminante no diferencia los dos grupos de salsas de mostaza.

En el análisis de correlación lineal, la relación más elevada se encuentra entre los ácidos palmítico y esteárico $(0,9591)$. El ácido erúcico presenta correlación negativa con el ácido linoléico, y el coeficiente de correlación es $-0,7739$ (Tabla IV).

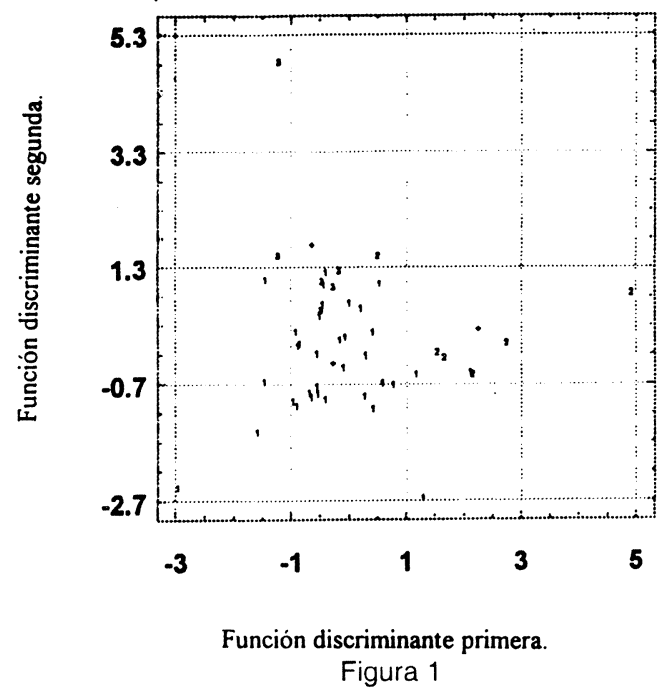

Análisis discrminante de las muestras de mostaza

1: Salsas finas de mostaza.

2: Mostaza (semilla y harina).

3: Salsas de mostaza con grano.

Tabla IV

Matriz de correlación de ácidos grasos

\begin{tabular}{|c|c|c|c|c|c|c|c|c|c|c|c|}
\hline & $16: 0$ & $19: 1$ & $18: 0$ & $18: 1$ & $18: 2$ & $18: 3$ & 20:1 & $20: 2$ & 22:0 & $22: 1$ & $24: 0$ \\
\hline \multicolumn{12}{|l|}{$16: 0$} \\
\hline $16: 1$ & 0,3572 & & & & & & & & & & \\
\hline $18: 0$ & 0,9591 & 0,2137 & & & & & & & & & \\
\hline $18: 1$ & 0,0430 & 0,3416 & $-0,0619$ & & & & & & & & \\
\hline $18: 2$ & 0,0819 & $-0,0899$ & 0,0457 & $-0,3334$ & & & & & & & \\
\hline $18: 3$ & $-0,1964$ & $-0,1744$ & $-0,1604$ & $-0,3676$ & 0,3281 & & & & & & \\
\hline $20: 1$ & $-0,2161$ & $-0,1880$ & $-0,2146$ & 0,3797 & 0,4727 & $-0,1246$ & & & & & \\
\hline $20: 2$ & $-0,0280$ & 0,0017 & $-0,0499$ & $-0,3414$ & 0,8835 & 0,1881 & 0,5094 & & & & \\
\hline 22:0 & $-0,1188$ & 0,0687 & $-0,1118$ & 0,1004 & 0,3126 & $-0,2116$ & 0,5202 & 0,5871 & & & \\
\hline 22:1 & $-0,1834$ & $-0,0709$ & $-0,1709$ & 0,6083 & $-0,7737$ & $-0,4912$ & 0,0661 & $-0,6871$ & $-0,0326$ & & \\
\hline $24: 0$ & $-0,0131$ & $-0,0996$ & $-0,0295$ & 0,1901 & $-0,0651$ & $-0,2404$ & 0,1839 & $-0,0477$ & 0,3126 & 0,2247 & \\
\hline
\end{tabular}




\section{AGRADECIMIENTOS}

A la Dra. Laura Coll Hellin por el asesoramiento en el estudio cromatográfico, y al Dr. Antonio Rueda Clausell por el tratamiento estadístico de los datos.

\section{BIBLIOGRAFÍA}

FAO (1991).— «Utilización de alimentos tropicales: semillas oleaginosas tropicales".-Organización de las Naciones Unidas para la Agricultura y la Alimentación, Roma.

Lebart, L. (1985).— «Tratamiento Estadístico de Datos: Métodos y Programas".-Barcelona: Marcombo Boixarem.

Ministerio de Sanidad y Consumo (1985).— «Análisis de Alimentos".-Métodos oficiales recomendados por el Centro de Investigaciones y Control de la Calidad.
Secretaría General para el Consumo. Dirección General de Control y Análisis de la Calidad. Ed. Servicio de Publicaciones Ministerio de Sanidad y Consumo.

Pearson, D. (1981).— «Técnicas de laboratorio para el análisis de alimentos".-Zaragoza: Acribia.

Karleskind, A. (1992). - "Manuel des corps gras. 1".París: Lavoisier.

Wolff, J.P. (1968).— “Manuel d'analyse des corps gras.».París: Azoulay.

Sindhu, K. T. C., Nagaraju, T. y Kantharas, U. K. (1993)."Glucosinolate and lipid composition of newer indian varieties of mustard and rapessed".-J. Food Sci. Technol, 30, 2, 137-138.

STSC (1986). — «Statgraphics User's Guide».-EE.UU.

Recibido: Julio 1998 Aceptado: Febrero 1999 